\title{
Study on a Stochastic Avian Influenza Epidemic Model with Generalized Incidence Rate
}

\author{
Shasha Ma \\ College of Science, University of Shanghai for Science and Technology, Shanghai, China \\ Email: mashasha3232@163.com
}

How to cite this paper: Ma, S.S. (2020) Study on a Stochastic Avian Influenza Epidemic Model with Generalized Incidence Rate. Open Journal of Applied Sciences, 10, 228-245.

https://doi.org/10.4236/ojapps.2020.105018

Received: April 11, 2020

Accepted: May 24, 2020

Published: May 27, 2020

Copyright (c) 2020 by author(s) and Scientific Research Publishing Inc. This work is licensed under the Creative Commons Attribution International License (CC BY 4.0).

http://creativecommons.org/licenses/by/4.0/ (c) (i) Open Access

\begin{abstract}
Avian Influenza, with a high mortality rate in human population, is considered to be one of the most significant potential threats to human beings. Based on a recent avian influenza SI-SIR model with logistic growth for birds, we propose a stochastic model with generalized incidence rate. For the stochastic avian-only system, sufficient conditions for the extinction of infected birds are established, and the existence of a unique ergodic stationary distribution is also obtained. For the stochastic avian-human system, a threshold number $R_{0}^{s}$ is established, and hence the extinction of disease is investigated. From the viewpoint of biology, the noise intensity in the infected birds plays a key role in the evolutionary dynamics. Moreover, we also analyze the asymptotic behavior around the endemic equilibrium of the corresponding deterministic model.
\end{abstract}

\section{Keywords}

Avian Influenza, Extinction, Ergodic Stationary Distribution, Asymptotic Behavior

\section{Introduction}

Avian Influenza, an acute infectious disease caused by influenza A virus, is a complicated disease that can not only infect poultry but also infect humans who have direct exposure to infected birds or contaminated environments. Because humans generally lack immunity to avian influenza virus, the disease has a high mortality rate. For instance, an outbreak of the avian influenza AH7N9 in China caused 134 cases with 45 deaths, from March 31 to August 31, 2013 [1]. According to the data reported by the World Health Organization (WHO), 860 human infections have been reported worldwide since 2003, with about half of those 
people dying [2]. Hence, the WHO considers the disease to be one of the most significant potential threats to human beings. To better analyze the spread of avian influenza, an increasing amount of research has been studied from the viewpoint of epidemiology and biomathematics. Alexander et al. [3] introduced the pathogenicity of four avian influenza viruses for chickens, turkeys and ducks. Grais et al. [4] and Ferguson et al. [5] took humans as the core and proposed some strategies to retard the spread of avian influenza in the population. Menach [6] regarded poultry farm as a unit to establish a model of infectious disease and focused on the transmission to humans. For more mathematical epidemic models on avian influenza, one can refer to [7] [8] [9] [10] and the references therein.

Recently, under the assumption that avian influenza virus does not spread from person to person and mutate, Liu et al. [1] proposed the following avian influenza bird-to-human transmission model

$$
\left\{\begin{array}{l}
\frac{\mathrm{d} S_{a}(t)}{\mathrm{d} t}=g\left(S_{a}\right)-\beta_{a} S_{a} I_{a} \\
\frac{\mathrm{d} I_{a}(t)}{\mathrm{d} t}=\beta_{a} S_{a} I_{a}-\left(\mu_{a}+\delta_{a}\right) I_{a} \\
\frac{\mathrm{d} S_{h}(t)}{\mathrm{d} t}=\Pi_{h}-\beta_{h} S_{h} I_{a}-\mu_{h} S_{h} \\
\frac{\mathrm{d} I_{h}(t)}{\mathrm{d} t}=\beta_{h} S_{h} I_{a}-\left(\mu_{h}+\delta_{h}+\gamma\right) I_{h} \\
\frac{\mathrm{d} R_{h}(t)}{\mathrm{d} t}=\gamma I_{h}-\mu_{h} R
\end{array}\right.
$$

where $S_{a}(t), I_{a}(t)$ denote the number of the susceptible and infective avian population (i.e. birds) at time $t$, respectively, $\beta_{a}$ is the transmission rate from infective birds to susceptible birds, $\mu_{a}$ is the natural death rate of the birds, $\delta_{a}$ is the disease-related death rate of the infected birds; $S_{h}(t), I_{h}(t)$ and $R_{h}(t)$ denote the number of the susceptible human, infective human and recovered human at time $t$, respectively, $\beta_{h}$ is the transmission rate from the infective birds to the susceptible human, $\mu_{h}$ is the natural death rate of the human population, $\delta_{h}$ is the disease-related death rate of the infected human; $\gamma$ is the recovery rate of the infective human. The function $g\left(S_{a}\right)$ describes the net growth rate of the avian population, and two cases are studied in the reference [1]. In the present article, we pick the case that the avian population is subject to the logistic growth, then $g\left(S_{a}\right)=r_{a} S_{a}\left(1-\frac{S_{a}}{k_{a}}\right)$, where $r_{a}$ is the intrinsic growth rate, $k_{a}$ is the maximal carrying capacity of the avian population.

The incidence rate of epidemic model plays a quite important role in describing the evolution of infectious disease, and system (1) chose bilinear ones, that is, $\beta_{a} S_{a} I_{a}$ and $\beta_{h} S_{h} I_{a}$. However, bilinear incidence rate becomes unrealistic when the number of the infective individual is getting larger [11]. Hence a large amount of research selects nonlinear incidence rates, such as saturated incidence 
rate of the form $\frac{\beta S I}{1+\alpha I}[11]$, and incidence rate with media coverage effect of the form $\beta \mathrm{e}^{-m I} S I$ [12]. In practice, the incidence function is frequently difficult to obtain because the details of disease transmission vary in different conditions. Therefore choosing generalized incidence rates may allow epidemic models to be more flexible in handling realistic data. For model (1), we replace $\beta_{a} S_{a} I_{a}$ with $\beta_{a} S_{a} f\left(I_{a}\right)$ to denote the generalized incidence rate between susceptible and infective birds, and replace $\beta_{h} S_{h} I_{a}$ with $\beta_{h} S_{h} G\left(I_{a}\right)$ to denote the generalized incidence rate between susceptible human and infective birds, then the model becomes

$$
\left\{\begin{array}{l}
\frac{\mathrm{d} S_{a}(t)}{\mathrm{d} t}=r_{a} S_{a}\left(1-\frac{S_{a}}{k_{a}}\right)-\beta_{a} S_{a} f\left(I_{a}\right) \\
\frac{\mathrm{d} I_{a}(t)}{\mathrm{d} t}=\beta_{a} S_{a} f\left(I_{a}\right)-\left(\mu_{a}+\delta_{a}\right) I_{a} \\
\frac{\mathrm{d} S_{h}(t)}{\mathrm{d} t}=\Pi_{h}-\beta_{h} S_{h} G\left(I_{a}\right)-\mu_{h} S_{h} \\
\frac{\mathrm{d} I_{h}(t)}{\mathrm{d} t}=\beta_{h} S_{h} G\left(I_{a}\right)-\left(\mu_{h}+\delta_{h}+\gamma\right) I_{h}
\end{array}\right.
$$

where the logistic function $g\left(S_{a}\right)=r_{a} S_{a}\left(1-\frac{S_{a}}{k_{a}}\right)$ is plugged in, and the recovered population is removed since it has no effect on the dynamics of susceptible population and infective population. Furthermore, the transmission of influenza is disturbed by various noises in the environment, such as the unpredictable contact with infected ones, population mobility and meteorological factors. It is shown that environment fluctuations have a important effect on the development of infectious disease [13] [14] [15]. For instance, Meng et al. [14] showed that a large stochastic disturbance can cause infectious diseases to go to extinction, and $\mathrm{Li}$ et al. [15] also found that the average number of infected individuals always with the increase of noise intensity.

These observations imply that stochastic disturbance is conductive to epidemic diseases control. Many researches choose white noise as an appropriate representation of environmental random fluctuations and study the effect of stochastic disturbance on the dynamics of epidemic models. Motivated by the approach in [16], we introduce Gaussian white noise which is directly proportional to $S_{a}(t), I_{a}(t), S_{h}(t)$ and $I_{h}(t)$, respectively, and finally arrive at the following stochastic model

$$
\left\{\begin{array}{l}
\mathrm{d} S_{a}(t)=\left[r_{a} S_{a}\left(1-\frac{S_{a}}{k_{a}}\right)-\beta_{a} S_{a} f\left(I_{a}\right)\right] \mathrm{d} t+\sigma_{1} S_{a} \mathrm{~d} B_{1}(t) \\
\mathrm{d} I_{a}(t)=\left[\beta_{a} S_{a} f\left(I_{a}\right)-\left(\mu_{a}+\delta_{a}\right) I_{a}\right] \mathrm{d} t+\sigma_{2} I_{a} \mathrm{~d} B_{2}(t) \\
\mathrm{d} S_{h}(t)=\left[\Pi_{h}-\beta_{h} S_{h} G\left(I_{a}\right)-\mu_{h} S_{h}\right] \mathrm{d} t+\sigma_{3} S_{h} \mathrm{~d} B_{3}(t) \\
\mathrm{d} I_{h}(t)=\left[\beta_{h} S_{h} G\left(I_{a}\right)-\left(\mu_{h}+\delta_{h}+\gamma\right) I_{h}\right] \mathrm{d} t+\sigma_{4} I_{h} \mathrm{~d} B_{4}(t)
\end{array}\right.
$$


where $B_{i}(t), i=1,2,3,4$, are independent standard Brownian motions with $B_{i}(0)=0$, and $\sigma_{i}>0(i=1,2,3,4)$ denote the intensities of white noise. Moreover, let $\left(\Omega, F,\left\{F_{t}\right\}_{t \geq 0}, \mathbb{P}\right)$ be a complete probability space with a filtration $\left\{F_{t}\right\}_{t \geq 0}$ satisfying the usual conditions (i.e., it is increasing and right continuous while $F_{0}$ contains all $\mathbb{P}$-null sets), then $B_{i}(t), i=1,2,3,4$ are defined on this complete probability space. We also introduce the notations:

$R_{+}^{n}=\left\{x=\left(x_{1}, x_{2}, \cdots, x_{n}\right) \in R_{n}: x_{i}>0, i=1,2, \cdots, n\right\}, a \wedge b=\min \{a, b\}$, $a \vee b=\max \{a, b\}$.

Throughout the paper, we further assume that

(H1) $f(\cdot): R_{+} \rightarrow R_{+}, G(\cdot): R_{+} \rightarrow R_{+}$and $f(0)=0, G(0)=0$, $0<f\left(I_{a}\right), G\left(I_{a}\right) \leq I_{a}$ hold for all $I_{a}>0$;

(H2) $h(x)$ is Lipschitz on $[0,+\infty)$, namely, there exists a constant $\theta>0$, such that $\left|h\left(x_{1}\right)-h\left(x_{2}\right)\right| \leq \theta\left|x_{1}-x_{2}\right|$ for any $x_{1}, x_{2} \in[0,+\infty)$, where $h(x)=f(x) / x$;

(H3) $f^{\prime}(0)=1, G^{\prime}(0)=1$, where $f^{\prime}(0), G^{\prime}(0)$ denote the derivative of the function $f(x), G(x)$ at $x=0$, respectively.

Our generalized incidence rates can be applied to some specific forms that have been frequently used, such as

(i) Linear type: $f\left(I_{a}\right)=I_{a}, G\left(I_{a}\right)=I_{a}$;

(ii) Saturated incidence rate: $f\left(I_{a}\right)=\frac{I_{a}}{1+\alpha I_{a}}, G\left(I_{a}\right)=\frac{I_{a}}{1+\alpha I_{a}^{2}}$.

The article is dedicated to investigating the dynamics of the stochastic avian influenza epidemic model (3). The rest of the paper is organized as follows. In the next section, the existence and uniqueness of positive solution is proved for system (3). In Section 3, we discuss the dynamics of the avian-only subsystem, and obtain the sufficient conditions for the extinction of the disease as well as the existence of an ergodic stationary distribution. In Section 4, the dynamics of the avian-human system are discussed, and the asymptotic behavior of system (3) around the unique endemic equilibrium of system (2) is also investigated.

\section{Dynamics of the Stochastic Avian-Only Subsystem}

Since $S_{a}, I_{a}, S_{h}$ and $I_{h}$ in system (3) denote the number of individuals, they should be nonnegative from the viewpoint of biology. We first introduce some basic definitions that will be used in the reminder of the article. In general, let $X(t)$ be a homogeneous Markov process in the d-dimension Euclidean space Rddescribed by the stochastic differential equation

$$
\mathrm{d} X(t)=f(X) \mathrm{d} t+\sum_{r=1}^{k} \sigma_{r}(X) \mathrm{d} B_{r}(t)
$$

then the diffusion matrix is defined as follows

$$
A(x)=\left(a_{i j}(x)\right), a_{i j}(x)=\sum_{r=1}^{k} \sigma_{r}^{i}(x) \sigma_{r}^{j}(x) .
$$

Furthermore, the differential operator $L$ is defined by 


$$
L V(x)=\sum_{i=1}^{d} f_{i}(x) \frac{\partial V(x)}{\partial x_{i}}+\frac{1}{2} \sum_{i, j=1}^{d} a_{i j}(x) \frac{\partial^{2} V(x)}{\partial x_{i} \partial x_{j}},
$$

where $V(x)$ is an arbitrary twice continuously differential real-value function.

To begin with, we present the following fundamental theorem which guarantees the existence and uniqueness of positive solution for system (3).

Theorem 2.1. For any initial value $\left(S_{a}(0), I_{a}(0), S_{h}(0), I_{h}(0)\right) \in R_{+}^{4}$, there is a unique positive solution $\left(S_{a}(t), I_{a}(t), S_{h}(t), I_{h}(t)\right)$ of system (3) on $t \geq 0$ and the solution will remain in $R_{+}^{4}$ with probability one, namely, $\left(S_{a}(t), I_{a}(t), S_{h}(t), I_{h}(t)\right) \in R_{+}^{4}$ for all $t \geq 0$ almost surely.

The proof is similar to those of [17] and hence is omitted.

Consider the stochastic avian-only subsystem as follows

$$
\left\{\begin{array}{l}
\mathrm{d} S_{a}(t)=\left(r_{a} S_{a}\left(1-\frac{S_{a}}{k_{a}}\right)-\beta_{a} S_{a} f\left(I_{a}\right)\right) \mathrm{d} t+\sigma_{1} S_{a} \mathrm{~d} B_{1}(t) \\
\mathrm{d} I_{a}(t)=\left(\beta_{a} S_{a} f\left(I_{a}\right)-\left(\mu_{a}+\delta_{a}\right) I_{a}\right) \mathrm{d} t+\sigma_{2} I_{a} \mathrm{~d} B_{2}(t)
\end{array}\right.
$$

It is obviously observed that the avian system is independent of the human system. Therefore in this section we will focus on the dynamics of the stochastic system (5), and our main goal is to discuss the extinction of disease and the existence of stationary distribution.

The threshold parameter $R_{0}$ of the deterministic system with respect to (5) can be computed by application of the next-generation matrix approach as

$$
R_{0}=\frac{\beta_{a} k_{a}}{\mu_{a}+\delta_{a}} .
$$

Motivated by the approach in [7], we introduce Gaussian white noise to $R_{0}$. Let

$$
R_{0}^{s}=\frac{\beta_{a} k_{a}}{\mu_{a}+\delta_{a}+\frac{\sigma_{2}^{2}}{2}} .
$$

Theorem 2.2. Let $\left(S_{a}(t), I_{a}(t)\right)$ be a solution of system (5) with any initial value $\left(S_{a}(0), I_{a}(0)\right) \in R_{+}^{2}$. If $R_{0}^{s}<1$, and $\sigma_{2}^{2}<2 r_{a}$ then

$$
\limsup _{t \rightarrow \infty} \frac{\ln I_{a}(t)}{t} \leq\left(\mu_{a}+\delta_{a}+\frac{\sigma_{2}^{2}}{2}\right)\left(R_{0}^{s}-1\right)<0,
$$

namely, $I_{a}(t)$ will tend to zero exponentially a.s. and the disease will tend to extinction with probability one.

The proof is similar to those of [17] and hence is omitted.

On the other hand, if we define

$$
\hat{R}_{0}=\frac{\beta_{a} \int_{0}^{\infty} x \pi(x) \mathrm{d} x}{\mu_{a}+\delta_{a}+\frac{\sigma_{2}^{2}}{2}},
$$

where $\pi(x)=Q x^{-2+\frac{2 r_{a}}{\sigma_{1}^{2}}} \mathrm{e}^{-\frac{2 r_{a} x}{\sigma_{1}^{2} k_{a}}}, x \in(0, \infty)$, then we will arrive at the following 
theorem which describes the extinction of the disease.

Theorem 2.3. If $\hat{R}_{0}<1$, then the solution $\left(S_{a}(t), I_{a}(t)\right)$ of system (5) satisfies

$$
\lim _{t \rightarrow \infty} I_{a}(t)=0 \text { a.s. }
$$

and the distribution of $S_{a}(t)$ converges weakly to the measure which has the density

$$
\pi(x)=Q x^{-2+\frac{2 r_{a}}{\sigma_{1}^{2}}} \mathrm{e}^{-\frac{2 r_{a} x}{\sigma_{1}^{2} k_{a}}}, \quad x \in(0, \infty),
$$

where $Q$ is a constant such that $\int_{0}^{\infty} \pi(x) \mathrm{d} x=1$.

Proof. Consider the following auxiliary logistic equation with random perturbation

$$
\mathrm{d} x(t)=x(t)\left(r_{a}-\frac{r_{a}}{k_{a}} x(t)\right) \mathrm{d} t+\sigma_{1} x(t) \mathrm{d} B_{1}(t), x(0)=S_{a}(0)>0,
$$

set $b(x)=r_{a} x\left(1-\frac{x}{k_{a}}\right), \sigma(x)=\sigma_{1} x, x \in(0, \infty)$, then we can obtain

$$
\int \frac{b(u)}{\sigma^{2}(u)} \mathrm{d} u=\frac{1}{\sigma_{1}^{2}} \int\left(\frac{r_{a}}{u}-\frac{r_{a}}{k_{a}}\right) \mathrm{d} u=\frac{r_{a}}{\sigma_{1}^{2}}\left(\ln u-\frac{u}{k_{a}}\right)+Q .
$$

Therefore,

$$
\mathrm{e}^{\int \frac{b(u)}{\sigma^{2}(u)} \mathrm{d} u}=\mathrm{e}^{Q+\ln u^{\frac{r_{a}}{\sigma_{1}^{2}}}-\frac{r_{a} u}{\sigma_{1}^{2} k_{a}}}=\mathrm{e}^{Q} u^{\frac{r_{a}}{\sigma_{1}^{2}}} \mathrm{e}^{-\frac{r_{a} u}{\sigma_{1}^{2} k_{a}}} .
$$

It is clear that

$$
\int_{0}^{\infty} \frac{1}{\sigma^{2}(x)} \mathrm{e}^{\int_{1}^{x} \frac{2 b(\tau)}{\sigma^{2}(\tau)} \mathrm{d} \tau} \mathrm{d} x=Q_{1} \int_{0}^{\infty} x^{-2+\frac{2 r_{a}}{\sigma_{1}^{2}}} \mathrm{e}^{-\frac{2 r_{a} x}{\sigma_{1}^{2} k_{a}}} \mathrm{~d} x<\infty
$$

where $Q_{1}=\frac{1}{\sigma_{1}^{2}} \mathrm{e}^{2 Q}$.

Consequently, the condition of Theorem 1.16 in [18] follows from (7). Thus system (6) has the ergodic property, and the invariant density is given by

$$
\pi(x)=Q x^{-2+\frac{2 r_{a}}{\sigma_{1}^{2}}} \mathrm{e}^{-\frac{2 r_{a} x}{\sigma_{1}^{2} k_{a}}}, x \in(0, \infty),
$$

where $Q$ is a constant such that $\int_{0}^{\infty} \pi(x) \mathrm{d} x=1$. From the ergodic theorem it follows that

$$
\lim _{t \rightarrow \infty} \frac{1}{t} \int_{0}^{\infty} x(s) \mathrm{d} s=\int_{0}^{\infty} x \pi(x) \mathrm{d} x \text { a.s. }
$$

Let $x(t)$ be the solution of (6) with the initial value $x(0)=S_{a}(0)>0$, then the comparison theorem of stochastic differential equation [19] yields

$$
S_{a}(t) \leq x(t) \text { a.s. }
$$

On the other hand, according to (9), we get 


$$
\begin{aligned}
\frac{\ln I_{a}(t)-\ln I_{a}(0)}{t} & \leq \frac{1}{t} \int_{0}^{t} \beta_{a} S_{a} \mathrm{~d} s-\left(\mu_{a}+\delta_{a}+\frac{\sigma_{2}^{2}}{2}\right)+\frac{\sigma_{2} B_{2}(t)}{t} \\
& \leq \frac{\beta_{a}}{t} \int_{0}^{t} x \mathrm{~d} s-\left(\mu_{a}+\delta_{a}+\frac{\sigma_{2}^{2}}{2}\right)+\frac{\sigma_{2} B_{2}(t)}{t} .
\end{aligned}
$$

Take the superior limit on both sides of (10), and note that $\hat{R}_{0}<1$, then

$$
\begin{aligned}
\limsup _{t \rightarrow \infty} \frac{I_{a}(t)}{t} & \leq \beta_{a} \int_{0}^{\infty} x \pi(x) \mathrm{d} x-\left(\mu_{a}+\delta_{a}+\frac{\sigma_{2}^{2}}{2}\right) \\
& =\left(\mu_{a}+\delta_{a}+\frac{\sigma_{2}^{2}}{2}\right)\left(\frac{\beta_{a} \int_{0}^{\infty} x \pi(x) \mathrm{d} x}{\mu_{a}+\delta_{a}+\frac{\sigma_{2}^{2}}{2}}-1\right) \\
& =\left(\mu_{a}+\delta_{a}+\frac{\sigma_{2}^{2}}{2}\right)\left(\hat{R}_{0}-1\right)<0 \text { a.s. }
\end{aligned}
$$

Which shows that $\lim _{t \rightarrow \infty} I_{a}(t)=0$ a.s.. Hence for any small $\varepsilon>0$, there exists a $t_{0}>0$ and a set $\stackrel{t \rightarrow \infty}{\Omega_{\varepsilon}} \subset \Omega$ Such that $\mathbb{P}\left(\Omega_{\varepsilon}\right)>1-\varepsilon$ and $\beta_{a} S_{a} f\left(I_{a}\right) \leq \varepsilon S_{a}$, for $t>t_{0}$ and $\omega \in \Omega_{\varepsilon}$. Hereon,

$$
\left[r_{a} S_{a}\left(1-\frac{S_{a}}{k_{a}}\right)-\varepsilon S_{a}\right] \mathrm{d} t+\sigma_{1} \mathrm{~d} B_{1}(t) \leq \mathrm{d} S_{a}(t) \leq\left[r_{a} S_{a}-\frac{r_{a} S_{a}^{2}}{k_{a}}\right] \mathrm{d} t+\sigma_{1} \mathrm{~d} B_{1}(t),
$$

it follows that the distribution of the process $S_{a}(t)$ converges weakly to the measure with the density $\pi$. This completes the proof.

We now concentrate on verifying the existence of an ergodic stationary distribution for system (5). The following lemma is fundamental in the paper.

Lemma 2.4. ([20]) The Markov process $X(t)$, the solution of system (4), has a unique ergodic stationary distribution $\pi(\cdot)$, if there exists a bounded domain $D \subset R^{l}$ with regular boundary $\Gamma$ and (B.1) there is a positive number $M$ such that $\sum_{i, j=1}^{l} a_{i j}(x) \xi_{i} \xi_{j} \geq M|\xi|^{2}, x=D, \xi \in R^{l}$, (B.2) there exists a nonnegative $C^{2}$-function $V$ such that $L V$ is negative for any $R^{l} \backslash D$. Then

$$
\mathbb{P}_{x}\left\{\lim _{t \rightarrow \infty} \frac{1}{T} \int_{0}^{T} f(X(t)) \mathrm{d} t=\int_{E_{l}} f(x) \mu(\mathrm{d} x)\right\}=1,
$$

for all $x \in R^{l}$, where $f(\cdot)$ is a function integrable with respect to the measure $\pi$.

Theorem 2.5. If $R_{0}^{s}>\frac{2 r_{a}}{2 r_{a}-\sigma_{1}^{2}}$ and $0<\sigma_{2}^{2}<2\left(\mu_{a}+\delta_{a}\right)$ hold, then for any initial value $\left(S_{a}(0), I_{a}(0)\right) \in R_{+}^{2}$, there exists a unique stationary distribution for system (5) and it has the ergodic property.

Proof. We have obtained that for any initial value $\left(S_{a}(0), I_{a}(0)\right) \in R_{+}^{2}$, there exists a unique global solution $\left(S_{a}(t), I_{a}(t)\right) \in R_{+}^{2}$. In order to prove Theorem 2.5, it suffices to verify the conditions (B.1) and (B.2) in Lemma 2.4. Firstly, we are trying to verify that there exists a neighborhood $D \in R_{+}^{2}$ and a nonnegative $C^{2}$-function $V$ such that for any $\left(S_{a}(t), I_{a}(t)\right) \in R_{+}^{2} \backslash D, L V$ is negative.

Define 


$$
h\left(S_{a}, I_{a}\right)=-C_{1}\left(\beta_{a} \ln S_{a}+\frac{r_{a}}{k_{a}} \ln I_{a}-\frac{\beta_{a}^{2}}{\mu_{a}+\delta_{a}} I_{a}\right)+\frac{\left(S_{a}+I_{a}\right)^{2}}{2},
$$

where

$$
C_{1}=\frac{2}{\lambda_{1}} \max \left\{2, \sup _{\left(S_{a}, I_{a}\right) \in R_{+}^{2}}\left\{-\frac{r_{a}}{2 S_{a}} S_{a}^{3}+\left(r_{a}+\frac{\sigma_{1}^{2}}{2}\right) S_{a}^{2}-\frac{\mu_{a}+\delta_{a}-\frac{\sigma_{2}^{2}}{2}}{2} I_{a}^{2}\right\}\right\} .
$$

And

$$
\lambda_{1}=\frac{r_{a}\left(\mu_{a}+\delta_{a}+\frac{\sigma_{2}^{2}}{2}\right)}{k_{a}}\left[\left(1-\frac{\sigma_{1}^{2}}{2 r_{a}}\right) R_{0}^{s}-1\right]>0 .
$$

It is standard to verify that $h\left(S_{a}, I_{a}\right)$ has a unique minimum value point $\left(\bar{S}_{a}, \bar{I}_{a}\right)$ in $R_{+}^{2}$.

Define

$$
V\left(S_{a}, I_{a}\right)=h\left(S_{a}, I_{a}\right)-h\left(\bar{S}_{a}, \bar{I}_{a}\right)=V_{1}+V_{2}-h\left(\bar{S}_{a}, \bar{I}_{a}\right) .
$$

where

$$
V_{1}=-C_{1}\left(\beta_{a} \ln S_{a}+\frac{r_{a}}{k_{a}} \ln I_{a}-\frac{\beta_{a}^{2}}{\mu_{a}+\delta_{a}} I_{a}\right), \quad V_{2}=\frac{\left(S_{a}+I_{a}\right)^{2}}{2} .
$$

Let $F\left(I_{a}\right):=1-\frac{f\left(I_{a}\right)}{I_{a}}$, due to the hypotheses (H2) and (H3), there exists a constant $\theta>0$, such that

$$
F\left(I_{a}\right)-F(0) \leq \theta\left(I_{a}-0\right),
$$

namely,

$$
1-\frac{f\left(I_{a}\right)}{I_{a}} \leq \theta I_{a} .
$$

According to inequality (14), we can obtain

$$
\begin{aligned}
L V_{1}= & -C_{1}\left[\beta_{a} r_{a}-\frac{\beta_{a} r_{a} S_{a}}{k_{a}}-\beta_{a}^{2} f\left(I_{a}\right)-\frac{\beta_{a} \sigma_{1}^{2}}{2}+\frac{\beta_{a} r_{a} S_{a} f\left(I_{a}\right)}{k_{a} I_{a}}\right. \\
& \left.-\frac{r_{a}}{k_{a}}\left(\mu_{a}+\delta_{a}+\frac{\sigma_{2}^{2}}{2}\right)-\frac{\beta_{a}^{3}}{\mu_{a}+\delta_{a}} f\left(I_{a}\right) S_{a}+I_{a}\right] \\
= & -C_{1}\left[\frac{r_{a}\left(\mu_{a}+\delta_{a}+\frac{\sigma_{2}^{2}}{2}\right)}{k_{a}}\left(\left(1-\frac{\sigma_{1}^{2}}{2 r_{a}}\right) R_{0}^{s}-1\right)-\frac{\beta_{a}^{3}}{\mu_{a}+\delta_{a}} f\left(I_{a}\right) S_{a}\right. \\
& \left.-\beta_{a}^{2}\left(f\left(I_{a}\right)-I_{a}\right)+\frac{\beta_{a} r_{a}}{k_{a}}\left(\frac{f\left(I_{a}\right)}{I_{a}}-1\right) S_{a}\right] \\
\leq & -C_{1} \lambda_{1}+C_{1} \frac{\beta_{a}^{3}}{\mu_{a}+\delta_{a}} f\left(I_{a}\right) S_{a}+C_{1} \frac{\theta \beta_{a} r_{a}}{k_{a}} S_{a} I_{a} \\
\leq & -C_{1} \lambda_{1}+\left(C_{1} \frac{\beta_{a}^{3}}{\mu_{a}+\delta_{a}}+C_{1} \frac{\theta \beta_{a} r_{a}}{k_{a}}\right) S_{a} I_{a} .
\end{aligned}
$$


Moreover,

$$
\begin{aligned}
L V_{2} & =\left(S_{a}+I_{a}\right)\left(r_{a} S_{a}-\frac{r_{a}}{k_{a}} S_{a}^{2}-\left(\mu_{a}+\delta_{a}\right) I_{a}\right)+\frac{\sigma_{1}^{2} S_{a}^{2}}{2}+\frac{\sigma_{2}^{2} I_{a}^{2}}{2} \\
& \leq-\frac{r_{a}}{k_{a}} S_{a}^{3}+\left(r_{a}+\frac{\sigma_{1}^{2}}{2}\right) S_{a}^{2}+\left|\mu_{a}+\delta_{a}-r_{a}\right| S_{a} I_{a}-\left(\mu_{a}+\delta_{a}-\frac{\sigma_{2}^{2}}{2}\right) I_{a}^{2}-\frac{r_{a}}{k_{a}} S_{a}^{2} I_{a} \\
& \leq-\frac{r_{a}}{k_{a}} S_{a}^{3}+\left(r_{a}+\frac{\sigma_{1}^{2}}{2}\right) S_{a}^{2}+\left|\mu_{a}+\delta_{a}-r_{a}\right| S_{a} I_{a}-\left(\mu_{a}+\delta_{a}-\frac{\sigma_{2}^{2}}{2}\right) I_{a}^{2} .
\end{aligned}
$$

Thus

$$
\begin{aligned}
L V= & L\left(V_{1}+V_{2}-h\left(\bar{S}_{a}, \bar{I}_{a}\right)\right) \\
\leq & -C_{1} \lambda_{1}+\left(C_{1} \frac{\beta_{a}^{3}}{\mu_{a}+\delta_{a}}+C_{1} \frac{\theta \beta_{a} r_{a}}{k_{a}}\right) S_{a} I_{a}-\frac{r_{a}}{k_{a}} S_{a}^{3} \\
& +\left(r_{a}+\frac{\sigma_{1}^{2}}{2}\right) S_{a}^{2}+\left|\mu_{a}+\delta_{a}-r_{a}\right| S_{a} I_{a}-\left(\mu_{a}+\delta_{a}-\frac{\sigma_{2}^{2}}{2}\right) I_{a}^{2} \\
= & -C_{1} \lambda_{1}-\frac{r_{a}}{k_{a}} S_{a}^{3}+\left(C_{1} \frac{\beta_{a}^{3}}{\mu_{a}+\delta_{a}}+C_{1} \frac{\theta \beta_{a} r_{a}}{k_{a}}+\left|\mu_{a}+\delta_{a}-r_{a}\right|\right) S_{a} I_{a} \\
& -\left(\mu_{a}+\delta_{a}-\frac{\sigma_{2}^{2}}{2}\right) I_{a}^{2}+\left(r_{a}+\frac{\sigma_{1}^{2}}{2}\right) S_{a}^{2} .
\end{aligned}
$$

Choose a sufficiently small $\varepsilon$ such that

$$
\begin{aligned}
0<\varepsilon<\left(C_{1} \frac{\beta_{a}^{3}}{\mu_{a}+\delta_{a}}+\frac{C_{1} \theta \beta_{a} r_{a}}{k_{a}}+\left|\mu_{a}+\delta_{a}-r_{a}\right|\right)^{-1} \min \left\{\frac{C_{1} \lambda_{1}}{4}, \frac{\mu_{a}+\delta_{a}-\frac{\sigma_{2}^{2}}{2}}{2}, \frac{r_{a}}{2 k_{a}}\right\}, \\
\min \left\{\frac{r_{a}}{2 k_{a} \varepsilon^{3}}, \frac{\mu_{a}+\delta_{a}-\frac{\sigma_{2}^{2}}{2}}{2}\right\} \geq 1-C_{1} \lambda_{1}+K_{1} .
\end{aligned}
$$

To confirm condition (B.2) of Lemma 2.4, we consider the bounded open set

$$
D_{\varepsilon}=\left\{\left(S_{a}, I_{a}\right) \in R_{+}^{2} \mid \varepsilon<S_{a}<\frac{1}{\varepsilon}, \varepsilon<I_{a}<\frac{1}{\varepsilon}\right\} .
$$

Define

$$
\begin{array}{ll}
D_{\varepsilon}^{1}=\left\{\left(S_{a}, I_{a}\right) \in R_{+}^{2} \mid 0<S_{a} \leq \varepsilon\right\}, & D_{\varepsilon}^{2}=\left\{\left(S_{a}, I_{a}\right) \in R_{+}^{2} \mid 0<I_{a} \leq \varepsilon\right\}, \\
D_{\varepsilon}^{3}=\left\{\left(S_{a}, I_{a}\right) \in R_{+}^{2} \mid S_{a} \geq \frac{1}{\varepsilon}\right\}, & D_{\varepsilon}^{4}=\left\{\left(S_{a}, I_{a}\right) \in R_{+}^{2} \mid I_{a} \geq \frac{1}{\varepsilon}\right\} .
\end{array}
$$

Obviously, $D_{\varepsilon}^{c}=D_{\varepsilon}^{1} \cup D_{\varepsilon}^{2} \cup D_{\varepsilon}^{3} \cup D_{\varepsilon}^{4}$. Next we will show that $L V \leq-1$ on $D_{\varepsilon}^{c}$.

Case 1. If $\left(S_{a}, I_{a}\right) \in D_{\varepsilon}^{1}$, then $S_{a} I_{a} \leq \varepsilon I_{a} \leq \varepsilon\left(1+I_{a}^{2}\right)$, and we have 


$$
\begin{aligned}
L V \leq & -C_{1} \lambda_{1}-\frac{r_{a}}{k_{a}} S_{a}^{3}+\left(C_{1} \frac{\beta_{a}^{3}}{\mu_{a}+\delta_{a}}+C_{1} \frac{\theta \beta_{a} r_{a}}{k_{a}}+\left|\mu_{a}+\delta_{a}-r_{a}\right|\right) S_{a} I_{a} \\
& -\left(\mu_{a}+\delta_{a}-\frac{\sigma_{2}^{2}}{2}\right) I_{a}^{2}+\left(r_{a}+\frac{\sigma_{1}^{2}}{2}\right) S_{a}^{2} \\
\leq & -\frac{C_{1} \lambda_{1}}{4}+\left(-\frac{C_{1} \lambda_{1}}{4}+\left(C_{1} \frac{\beta_{a}^{3}}{\mu_{a}+\delta_{a}}+C_{1} \frac{\theta \beta_{a} r_{a}}{k_{a}}+\left|\mu_{a}+\delta_{a}-r_{a}\right|\right) \varepsilon\right) \\
& +\left(-\frac{\mu_{a}+\delta_{a}-\frac{\sigma_{2}^{2}}{2}}{2}+\left(C_{1} \frac{\beta_{a}^{3}}{\mu_{a}+\delta_{a}}+C_{1} \frac{\theta \beta_{a} r_{a}}{k_{a}}+\left|\mu_{a}+\delta_{a}-r_{a}\right|\right) \varepsilon\right) I_{a}^{2} \\
& +\left(-\frac{C_{1} \lambda_{1}}{2}+\left(r_{a}+\frac{\sigma_{1}^{2}}{2}\right) S_{a}^{2}-\frac{r_{a}}{2 k_{a}} S_{a}^{3}-\frac{\mu_{a}+\delta_{a}-\frac{\sigma_{2}^{2}}{2}}{2} I_{a}^{2}\right),
\end{aligned}
$$

where $L V=L\left(V_{1}+V_{2}-h\left(\bar{S}_{a}, \bar{I}_{a}\right)\right)$. According to the definition of $C_{1}$ and Inequality (15), we can obtain that $L V \leq-\frac{C_{1} \lambda_{1}}{4} \leq-1$ on $D_{\varepsilon}^{1}$.

Case 2. If $\left(S_{a}, I_{a}\right) \in D_{\varepsilon}^{2}$, then $S_{a} I_{a} \leq \varepsilon S_{a} \leq \varepsilon\left(1+S_{a}^{3}\right)$, and we have

$$
\begin{aligned}
L V \leq & -\frac{C_{1} \lambda_{1}}{4}+\left(-\frac{C_{1} \lambda_{1}}{4}+\left(C_{1} \frac{\beta_{a}^{3}}{\mu_{a}+\delta_{a}}+C_{1} \frac{\theta \beta_{a} r_{a}}{k_{a}}+\left|\mu_{a}+\delta_{a}-r_{a}\right|\right) \varepsilon\right) \\
& +\left[-\frac{r_{a}}{2 k_{a}}+\left(C_{1} \frac{\beta_{a}^{3}}{\mu_{a}+\delta_{a}}+C_{1} \frac{\theta \beta_{a} r_{a}}{k_{a}}+\left|\mu_{a}+\delta_{a}-r_{a}\right|\right) \varepsilon\right] S_{a}^{3} \\
& +\left(-\frac{C_{1} \lambda_{1}}{2}+\left(r_{a}+\frac{\sigma_{1}^{2}}{2}\right) S_{a}^{2}-\frac{r_{a}}{2 k_{a}} S_{a}^{3}-\frac{\mu_{a}+\delta_{a}-\frac{\sigma_{2}^{2}}{2}}{2} I_{a}^{2}\right) .
\end{aligned}
$$

where $L V=L\left(V_{1}+V_{2}-h\left(\bar{S}_{a}, \bar{I}_{a}\right)\right)$. By virtute of the definition of $C_{1}$ and Inequality (15), we can obtain that $L V \leq-\frac{C_{1} \lambda_{1}}{4} \leq-1$ on $D_{\varepsilon}^{2}$.

Case 3. If $\left(S_{a}, I_{a}\right) \in D_{\varepsilon}^{3}$, then we have $S_{a} I_{a} \leq \frac{2}{5} S_{a}^{\frac{5}{2}}+\frac{3}{5} I_{a}^{\frac{5}{3}}$ by Young inequality.

Hence,

$$
\begin{aligned}
L V \leq & -C_{1} \lambda_{1}-\frac{r_{a}}{k_{a}} S_{a}^{3}+\left[\left(C_{1} \frac{\beta_{a}^{3}}{\mu_{a}+\delta_{a}}+C_{1} \frac{\theta \beta_{a} r_{a}}{k_{a}}+\left|\mu_{a}+\delta_{a}-r_{a}\right|\right)\left(\frac{2}{5} S_{a}^{\frac{5}{2}}+\frac{3}{5} I_{a}^{\frac{5}{3}}\right)\right. \\
& \left.-\frac{r_{a}}{2 k_{a}} S_{a}^{3}+\left(r_{a}+\frac{\sigma_{1}^{2}}{2}\right) S_{a}^{2}-\frac{\mu_{a}+\delta_{a}-\frac{\sigma_{2}^{2}}{2}}{2} I_{a}^{2}\right]-\frac{\mu_{a}+\delta_{a}-\frac{\sigma_{2}^{2}}{2}}{2} I_{a}^{2} \\
& \leq-C_{1} \lambda_{1}-\frac{r_{a}}{2 k_{a} \varepsilon^{3}}+K_{1},
\end{aligned}
$$


where $L V=L\left(V_{1}+V_{2}-h\left(\bar{S}_{a}, \bar{I}_{a}\right)\right)$,

$$
\begin{aligned}
K_{1}= & \max _{\left(S_{a}, I_{a}\right) \in(0,+\infty)}\left\{\left(C_{1} \frac{\beta_{a}^{3}}{\mu_{a}+\delta_{a}}+C_{1} \frac{\theta \beta_{a} r_{a}}{k_{a}}+\left|\mu_{a}+\delta_{a}-r_{a}\right|\right)\left(\frac{2}{5} S_{a}^{\frac{5}{2}}+\frac{3}{5} I_{a}^{\frac{5}{3}}\right)\right. \\
& \left.-\frac{r_{a}}{2 k_{a}} S_{a}^{3}+\left(r_{a}+\frac{\sigma_{1}^{2}}{2}\right) S_{a}^{2}-\frac{\mu_{a}+\delta_{a}-\frac{\sigma_{2}^{2}}{2}}{2} I_{a}^{2}\right\} .
\end{aligned}
$$

According to Inequality (16) we can obtain that $L V \leq-1$ on $D_{\varepsilon}^{3}$.

Case 4. If $\left(S_{a}, I_{a}\right) \in D_{\varepsilon}^{4}$, similar to the case 3 , it is easy to get

$$
L V \leq-C_{1} \lambda_{1}-\frac{\mu_{a}+\delta_{a}-\frac{\sigma_{2}^{2}}{2}}{2} I_{a}^{2}+K_{1} \leq-C_{1} \lambda_{1}-\frac{\mu_{a}+\delta_{a}-\frac{\sigma_{2}^{2}}{2}}{2} \varepsilon^{2}+K_{1} \leq-1 .
$$

According to inequality (16), we obtain that $L V \leq-1$ on $D_{\varepsilon}^{4}$. That is, the condition (B.2) holds.

The diffusion matrix of system (5) is given by

$$
A=\left(\begin{array}{cc}
\sigma_{1}^{2} S_{a}^{2} & 0 \\
0 & \sigma_{2}^{2} I_{a}^{2}
\end{array}\right)
$$

Choose $M=\min _{\left(S_{a}, I_{a}\right) \in \bar{D} \subset R_{+}^{2}}\left\{\sigma_{1}^{2} S_{a}^{2}, \sigma_{2}^{2} I_{a}^{2}\right\}$, we have

$$
\sum_{i, j=1}^{2} a_{i, j}\left(S_{a}, I_{a}\right) \xi_{i} \xi_{j}=\sigma_{1}^{2} S_{a}^{2} \xi_{i}^{2}+\sigma_{2}^{2} I_{a}^{2} \xi_{j}^{2} \geq M|\xi|^{2},\left(S_{a}, I_{a}\right) \in \bar{D}, \xi=\left(\xi_{i}, \xi_{j}\right) \in R_{+}^{2} .
$$

Then the condition (B.1) in Lemma 2.4 holds. Consequently, system (5) has a unique stationary distribution and it is ergodic. The proof is completed.

From the biological perspective, stationary distribution can be considered as a weak stability of the system, and the ergodicity tells us that it is persistent in the time average. Moreover, under the assumption $\sigma_{1}^{2}<2 r_{a}$, the condition in the above theorem implies $R_{0}^{s}>\frac{2 r_{a}}{2 r_{a}-\sigma_{1}^{2}}>1$. It should be mentioned that the long time behavior is remained to be unknown if $1<R_{0}^{s}<\frac{2 r_{a}}{2 r_{a}-\sigma_{1}^{2}}$. The observation also indicates that it is difficult to obtain a threshold number for system (5), because of the logistic growth rate in the avian population.

\section{The Asymptotic Behavior of Stochastic Full System}

For any initial value $\left(S_{a}(0), I_{a}(0), S_{h}(0), I_{h}(0)\right) \in R_{+}^{4}$, system (3) has a unique positive solution $\left(S_{a}(t), I_{a}(t), S_{h}(t), I_{h}(t)\right)$ on $t \geq 0$, and the solution will remain in $R_{+}^{4}$ with probability one. In this section, we will investigate the dynamical behavior of the stochastic full system. From analysis of Section 3, we can easily deduce the following result.

Theorem 3.1. If $R_{0}^{s}<1$, then for any initial value 
$\left(S_{a}(0), I_{a}(0), S_{h}(0), I_{h}(0)\right) \in R_{+}^{4}$, the disease of system (3) will tend to extinction, almost surely.

The proof is similar to those of theorem 2.3 and hence is omitted.

If the deterministic system (2) has an endemic equilibrium, then it means the disease will persist in the long term. Since stochastic system does not exist endemic equilibrium, it is interesting to investigate the asymptotic behavior of global positive solution of system (3) around endemic equilibrium.

Lemma 3.2. Suppose that the function $\frac{x}{f(x)}$ is monotone increasing on $(0,+\infty)$, then there exists a unique endemic equilibrium for system (2) if $1<R_{0}<2$.

Proof. An endemic equilibrium $E^{*}$ of system (2) satisfie

$$
\left\{\begin{array}{l}
r_{a} S_{a}^{*}-\frac{r_{a}}{k_{a}} S_{a}^{* 2}-\beta_{a} S_{a}^{*} f\left(I_{a}^{*}\right)=0 \\
\beta_{a} S_{a}^{*} f\left(I_{a}^{*}\right)-\left(\mu_{a}+\delta_{a}\right) I_{a}^{*}=0 \\
\Pi_{h}-\beta_{h} S_{h}^{*} G\left(I_{a}^{*}\right)-\mu_{h} S_{h}^{*}=0 \\
\beta_{h} S_{h}^{*} G\left(I_{a}^{*}\right)-\left(\mu_{h}+\delta_{h}+\gamma\right) I_{h}^{*}=0 .
\end{array}\right.
$$

Furthermore,

$$
\begin{aligned}
& S_{a}^{*}=\frac{\left(\mu_{a}+\delta_{a}\right) I_{a}^{*}}{\beta_{a} f\left(I_{a}^{*}\right)}, S_{h}^{*}=\frac{\Pi_{h}}{\mu_{h}+\beta_{h} G\left(I_{a}^{*}\right)}, \\
& I_{h}^{*}=\frac{\beta_{h} S_{h}^{*} G\left(I_{a}^{*}\right)}{\mu_{h}+\delta_{h}+\gamma}=\frac{\beta_{h} \Pi_{h} G\left(I_{a}^{*}\right)}{\left(\mu_{h}+\delta_{h}+\gamma\right)\left(\mu_{h}+\beta_{h} G\left(I_{a}^{*}\right)\right)},
\end{aligned}
$$

and

$$
\frac{r_{a}\left(\mu_{a}+\delta_{a}\right) I_{a}^{*}}{\beta_{a} f\left(I_{a}^{*}\right)}-\frac{r_{a}\left(\mu_{a}+\delta_{a}\right)^{2}}{k_{a} \beta_{a}^{2}}\left(\frac{I_{a}^{*}}{f\left(I_{a}^{*}\right)}\right)^{2}-\left(\mu_{a}+\delta_{a}\right) I_{a}^{*}=0 .
$$

Set

$$
\begin{aligned}
H\left(I_{a}\right) & :=\frac{r_{a}\left(\mu_{a}+\delta_{a}\right) I_{a}}{\beta_{a} f\left(I_{a}\right)}-\frac{r_{a}\left(\mu_{a}+\delta_{a}\right)^{2}}{k_{a} \beta_{a}^{2}}\left(\frac{I_{a}}{f\left(I_{a}\right)}\right)^{2}-\left(\mu_{a}+\delta_{a}\right) I_{a} \\
& =-\frac{r_{a}\left(\mu_{a}+\delta_{a}\right)^{2}}{\beta_{a}^{2} k_{a}}\left(\frac{I_{a}}{f\left(I_{a}\right)}-\frac{R_{0}}{2}\right)^{2}+\frac{r_{a} k_{a}}{4}-\left(\mu_{a}+\delta_{a}\right) I_{a},
\end{aligned}
$$

Recall that $I_{a} / f\left(I_{a}\right) \geq 1$, and $\lim _{t \rightarrow \infty} \frac{I_{a}}{f\left(I_{a}\right)}=\frac{1}{f^{\prime}(0)}=1$, then $\lim _{t \rightarrow \infty} H\left(I_{a}\right)>0$ if $R_{0}>1$. Since the function $x / f(x)$ is monotone increasing on $(0,+\infty)$, $H\left(I_{a}\right)$ has a unique positive zero point $I_{a}^{*}$ if $1<R_{0}<2$. Hence, system (2) has a unique endemic equilibrium $E^{*}=\left(S_{a}^{*}, I_{a}^{*}, S_{h}^{*}, I_{h}^{*}\right)$. The proof is completed.

Theorem 3.3. Suppose the conditions in Lemma 3.2 hold, then system (2) possesses a unique endemic equilibrium $E^{*}=\left(S_{a}^{*}, I_{a}^{*}, S_{h}^{*}, I_{h}^{*}\right)$. Let 
$\left(S_{a}(t), I_{a}(t), S_{h}(t), I_{h}(t)\right)$ be the solution of system (3) with any initial value $\left(S_{a}(0), I_{a}(0), S_{h}(0), I_{h}(0)\right) \in R_{+}^{4}$. If $m_{1}:=\frac{r_{a}}{k_{a}}-\frac{\sqrt{3}+2}{\sqrt{3}} \beta_{a}>0$,

$m_{2}:=\mu_{a}+\delta_{a}-\sigma_{2}^{2}-\frac{\sqrt{3}+4}{\sqrt{3}} \beta_{a}-\beta_{h}\left(2 \mu_{h}+\delta_{h}+\gamma\right)>0 \quad$,

$m_{3}:=\frac{\beta_{h} G\left(I_{a}^{*}\right)}{I_{h}^{*}}\left(\mu_{h}-\sigma_{3}^{2}\right)-2 \beta_{h}\left(2 \mu_{h}+\delta_{h}+\gamma\right)>0$ and

$m_{4}:=\frac{\beta_{h} G\left(I_{a}^{*}\right)}{I_{h}^{*}}\left(\mu_{h}+\delta_{h}+\gamma-\sigma_{4}^{2}\right)>0$

hold, then

$\limsup _{t \rightarrow \infty} \frac{1}{t} E \int_{0}^{t}\left(m_{1}\left(S_{a}(s)-S_{a}^{*}\right)^{2}+m_{2}\left(I_{a}(s)-I_{a}^{*}\right)^{2}+m_{3}\left(S_{h}(s)-S_{h}^{*}\right)^{2}+m_{4}\left(I_{h}(s)-I_{h}^{*}\right)^{2}\right) \mathrm{d} s \leq \eta$,

where

$$
\begin{aligned}
\eta= & \frac{\beta_{a} f^{2}\left(I_{a}^{*}\right)}{2}+\left(3 \beta_{a}+\sigma_{2}^{2}\right) I_{a}^{* 2}+\left(\frac{3 \beta_{a}}{2}+\frac{\sigma_{1}^{2}}{2}\right) S_{a}^{*}+\beta_{a} S_{a}^{*} I_{a}^{*} f\left(I_{a}^{*}\right) \\
& +\left(\sigma_{3}^{2} S_{h}^{* 2}+\sigma_{4}^{2} I_{h}^{* 2}\right) \frac{\beta_{h} G\left(I_{a}^{*}\right)}{I_{h}^{*}}+\left(2 \beta_{h} S_{h}^{*}+\beta_{h} I_{a}^{* 2}+\frac{\sigma_{3}^{2} I_{h}^{*}}{2}\right)\left(2 \mu_{h}+\delta_{h}+\gamma\right) .
\end{aligned}
$$

Proof. Define $C^{2}$ functions as follows

$$
\begin{aligned}
& V_{1}=S_{a}-S_{a}^{*}-S_{a}^{*} \ln \frac{S_{a}}{S_{a}^{*}}, \quad V_{2}=\frac{\left(I_{a}-I_{a}^{*}\right)^{2}}{2}, \\
& V_{3}=\frac{\left(S_{h}-S_{h}^{*}+I_{h}-I_{h}^{*}\right)^{2}}{2}, V_{4}=I_{h}-I_{h}^{*}-I_{h}^{*} \ln \frac{I_{h}}{I_{h}^{*}} .
\end{aligned}
$$

Using the Itô's formula, we have

$$
\begin{aligned}
L V_{1}= & \frac{S_{a}-S_{a}^{*}}{S_{a}}\left(r_{a} S_{a}-\frac{r_{a}}{k_{a}} S_{a}^{2}-\beta_{a} S_{a} f\left(I_{a}\right)\right)+\frac{\sigma_{1}^{2}}{2} S_{a}^{*} \\
= & \left(S_{a}-S_{a}^{*}\right)\left(r_{a}-\frac{r_{a}}{k_{a}} S_{a}^{*}-\beta_{a} f\left(I_{a}\right)\right)+\frac{\sigma_{1}^{2} S_{a}^{*}}{2} \\
= & \left(S_{a}-S_{a}^{*}\right)\left(\frac{r_{a}}{k_{a}} S_{a}^{*}+\beta_{a} f\left(I_{a}^{*}\right)-\frac{r_{a}}{k_{a}} S_{a}-\beta_{a} f\left(I_{a}\right)\right)+\frac{\sigma_{1}^{2} S_{a}^{*}}{2} \\
= & -\frac{r_{a}}{k_{a}}\left(S_{a}-S_{a}^{*}\right)^{2}+\beta_{a} S_{a} f\left(I_{a}^{*}\right)-\beta_{a} S_{a} f\left(I_{a}\right) \\
& +\beta_{a} S_{a}^{*} f\left(I_{a}\right)-\beta_{a} S_{a}^{*} f\left(I_{a}^{*}\right)+\frac{\sigma_{1}^{2} S_{a}^{*}}{2} \\
\leq & -\frac{r_{a}}{k_{a}}\left(S_{a}-S_{a}^{*}\right)^{2}+\beta_{a} S_{a} f\left(I_{a}^{*}\right)+\beta_{a} S_{a}^{*} f\left(I_{a}\right)+\frac{\sigma_{1}^{2} S_{a}^{*}}{2} \\
\leq & -\frac{r_{a}}{k_{a}}\left(S_{a}-S_{a}^{*}\right)^{2}+\beta_{a} S_{a} f\left(I_{a}^{*}\right)+\beta_{a} S_{a}^{*} I_{a}+\frac{\sigma_{1}^{2} S_{a}^{*}}{2} \\
\leq & -\frac{r_{a}}{k_{a}}\left(S_{a}-S_{a}^{*}\right)^{2}+\beta_{a} \frac{S_{a}^{2}+f^{2}\left(I_{a}^{*}\right)}{2}+\beta_{a} \frac{S_{a}^{* 2}+I_{a}^{2}}{2}+\frac{\sigma_{1}^{2} S_{a}^{*}}{2}
\end{aligned}
$$




$$
\begin{aligned}
\leq & -\frac{r_{a}}{k_{a}}\left(S_{a}-S_{a}^{*}\right)^{2}+\beta_{a}\left(S_{a}-S_{a}^{*}\right)^{2}+\beta_{a}\left(I_{a}-I_{a}^{*}\right)^{2} \\
& +\frac{\beta_{a} f^{2}\left(I_{a}^{*}\right)}{2}+\frac{3 \beta_{a} S_{a}^{* 2}}{2}+\frac{\sigma_{1}^{2} S_{a}^{*}}{2}+\beta_{a} I_{a}^{* 2} \\
\leq & -\left(\frac{r_{a}}{k_{a}}-\beta_{a}\right)\left(S_{a}-S_{a}^{*}\right)^{2}+\beta_{a}\left(I_{a}-I_{a}^{*}\right)^{2}+\eta_{1}, \\
L V_{2}= & \left(I_{a}-I_{a}^{*}\right)\left(\beta_{a} S_{a} f\left(I_{a}\right)-\left(\mu_{a}+\delta_{a}\right) I_{a}\right)+\frac{\sigma_{2}^{2} I_{a}^{2}}{2} \\
\leq & \left(I_{a}-I_{a}^{*}\right)\left(\beta_{a} S_{a} f\left(I_{a}\right)-\left(\mu_{a}+\delta_{a}\right) I_{a}^{*}+\left(\mu_{a}+\delta_{a}\right) I_{a}^{*}-\left(\mu_{a}+\delta_{a}\right) I_{a}\right) \\
& +\sigma_{2}^{2}\left(I_{a}-I_{a}^{*}\right)^{2}+\sigma_{2}^{2} I_{a}^{* 2} \\
= & \beta_{a} S_{a} I_{a} f\left(I_{a}\right)+\beta_{a} S_{a}^{*} I_{a}^{*} f\left(I_{a}^{*}\right)-\beta_{a} S_{a} I_{a}^{*} f\left(I_{a}\right)-\beta_{a} S_{a}^{*} I_{a} f\left(I_{a}^{*}\right) \\
& -\left(\mu_{a}+\delta_{a}-\sigma_{2}^{2}\right)\left(I_{a}-I_{a}^{*}\right)^{2}+\sigma_{2}^{2} I_{a}^{* 2} \\
\leq & \beta_{a} S_{a} I_{a}^{2}-\left(\mu_{a}+\delta_{a}-\sigma_{2}^{2}\right)\left(I_{a}-I_{a}^{*}\right)^{2}+\sigma_{2}^{2} I_{a}^{* 2}+\beta_{a} S_{a}^{*} I_{a}^{*} f\left(I_{a}^{*}\right) \\
\leq & \beta_{a} \frac{S_{a}^{2}+2 I_{a}^{2}}{\sqrt{3}}-\left(\mu_{a}+\delta_{a}-\sigma_{2}^{2}\right)\left(I_{a}-I_{a}^{*}\right)^{2}+\sigma_{2}^{2} I_{a}^{* 2}+\beta_{a} S_{a}^{*} I_{a}^{*} f\left(I_{a}^{*}\right) \\
\leq & \frac{2 \beta_{a}}{\sqrt{3}}\left(S_{a}-S_{a}^{*}\right)^{2}-\left(\mu_{a}+\delta_{a}-\sigma_{2}^{2}-\frac{4 \beta_{a}}{\sqrt{3}}\right)\left(I_{a}-I_{a}^{*}\right)^{2} \\
+ & \left(\sigma_{2}^{2}+\frac{4 \beta_{a}}{\sqrt{3}} I_{a}^{* 2}+\beta_{a} S_{a}^{*} I_{a}^{*} f\left(I_{a}^{*}\right)+\frac{2 \beta_{a}}{\sqrt{3}} S_{a}^{* 2}\right. \\
\leq & \frac{2 \beta_{a}}{\sqrt{3}}\left(S_{a}-S_{a}^{*}\right)^{2}-\left(\mu_{a}+\delta_{a}-\sigma_{2}^{2}-\frac{4 \beta_{a}}{\sqrt{3}}\right)\left(I_{a}-I_{a}^{*}\right)^{2}+\eta_{2},
\end{aligned}
$$

where the basic inequality $x y z \leq \frac{x^{2}+y^{2}+z^{2}}{\sqrt{3}}\left(x, y, z \in R_{+}\right)$is used. Similarly,

$$
\begin{aligned}
L V_{3}= & \left(S_{h}-S_{h}^{*}+I_{h}-I_{h}^{*}\right)\left(\Pi_{h}-\mu_{h} S_{h}-\left(\mu_{h}+\delta_{h}+\gamma\right) I_{h}\right)+\frac{\sigma_{3}^{2} S_{h}^{2}+\sigma_{4}^{2} I_{h}^{2}}{2} \\
\leq & \left(S_{h}-S_{h}^{*}+I_{h}-I_{h}^{*}\right)\left[\mu_{h} S_{h}^{*}+\left(\mu_{h}+\delta_{h}+\gamma\right) I_{h}^{*}-\mu_{h} S_{h}-\left(\mu_{h}+\delta_{h}+\gamma\right) I_{h}\right] \\
& +\sigma_{3}^{2}\left(S_{h}-S_{h}^{*}\right)^{2}+\sigma_{4}^{2}\left(I_{h}-I_{h}^{*}\right)^{2}+\sigma_{3}^{2} S_{h}^{* 2}+\sigma_{4}^{2} I_{h}^{* 2} \\
= & \left(S_{h}-S_{h}^{*}+I_{h}-I_{h}^{*}\right)\left[\mu_{h}\left(S_{h}^{*}-S_{h}\right)+\left(\mu_{h}+\delta_{h}+\gamma\right)\left(I_{h}^{*}-I_{h}\right)\right] \\
& +\sigma_{3}^{2}\left(S_{h}-S_{h}^{*}\right)^{2}+\sigma_{4}^{2}\left(I_{h}-I_{h}^{*}\right)^{2}+\sigma_{3}^{2} S_{h}^{* 2}+\sigma_{4}^{2} I_{h}^{* 2} \\
= & -\mu_{h}\left(S_{h}-S_{h}^{*}\right)^{2}+\left(\mu_{h}+\delta_{h}+\gamma\right)\left(S_{h}-S_{h}^{*}\right)\left(I_{h}^{*}-I_{h}\right) \\
& +\mu_{h}\left(S_{h}-S_{h}^{*}\right)\left(I_{h}^{*}-I_{h}\right)-\left(\mu_{h}+\delta_{h}+\gamma\right)\left(I_{h}-I_{h}^{*}\right)^{2} \\
& +\sigma_{3}^{2}\left(S_{h}-S_{h}^{*}\right)^{2}+\sigma_{4}^{2}\left(I_{h}-I_{h}^{*}\right)^{2}+\sigma_{3}^{2} S_{h}^{* 2}+\sigma_{4}^{2} I_{h}^{* 2} \\
= & -\left(\mu_{h}-\sigma_{3}^{2}\right)\left(S_{h}-S_{h}^{*}\right)^{2}-\left(2 \mu_{h}+\delta_{h}+\gamma\right)\left(S_{h}-S_{h}^{*}\right)\left(I_{h}-I_{h}^{*}\right) \\
& -\left(\mu_{h}+\delta_{h}+\gamma-\sigma_{4}^{2}\right)\left(I_{h}-I_{h}^{*}\right)^{2}+\eta_{3},
\end{aligned}
$$

And 


$$
\begin{aligned}
L V_{4}= & \frac{I_{h}-I_{h}^{*}}{I_{h}}\left(\beta_{h} S_{h} G\left(I_{a}\right)-\left(\mu_{h}+\delta_{h}+\gamma\right) I_{h}\right)+\frac{\sigma_{4}^{2} I_{h}^{*}}{2} \\
= & \left(I_{h}-I_{h}^{*}\right)\left(\frac{\beta_{h} S_{h} G\left(I_{a}\right)}{I_{h}}-\left(\mu_{h}+\delta_{h}+\gamma\right)\right)+\frac{\sigma_{4}^{2} I_{h}^{*}}{2} \\
= & \beta_{h} S_{h}\left(I_{h}-I_{h}^{*}\right)\left(\frac{G\left(I_{a}\right)}{I_{h}}-\frac{G\left(I_{a}^{*}\right)}{I_{h}^{*}}\right) \\
& +\beta_{h} \frac{G\left(I_{a}^{*}\right)}{I_{h}^{*}}\left(I_{h}-I_{h}^{*}\right)\left(S_{h}-S_{h}^{*}\right)+\frac{\sigma_{4}^{2} I_{h}^{*}}{2} \\
= & \beta_{h} S_{h} G\left(I_{a}\right)-\frac{\beta_{h} S_{h} I_{h} G\left(I_{a}^{*}\right)}{I_{h}^{*}}-\frac{\beta_{h} S_{h} I_{h}^{*} G\left(I_{a}\right)}{I_{h}}+\beta_{h} S_{h} G\left(I_{a}^{*}\right) \\
& +\frac{\beta_{h} G\left(I_{a}^{*}\right)}{I_{h}^{*}}\left(I_{h}-I_{h}^{*}\right)\left(S_{h}-S_{h}^{*}\right)+\frac{\sigma_{4}^{2} I_{h}^{*}}{2},
\end{aligned}
$$

due to the hypotheses (H1)

$$
\begin{aligned}
L V_{4} \leq & \beta_{h} S_{h} I_{a}+\beta_{h} S_{h} G\left(I_{a}^{*}\right)+\frac{\beta_{h} G\left(I_{a}^{*}\right)}{I_{h}^{*}}\left(I_{h}-I_{h}^{*}\right)\left(S_{h}-S_{h}^{*}\right)+\frac{\sigma_{4}^{2} I_{h}^{*}}{2} \\
\leq & \beta_{h} \frac{S_{h}^{2}+I_{a}^{2}}{2}+\beta_{h} \frac{S_{h}^{2}+G^{2}\left(I_{a}^{*}\right)}{2}+\frac{\beta_{h} G\left(I_{a}^{*}\right)}{I_{h}^{*}}\left(I_{h}-I_{h}^{*}\right)\left(S_{h}-S_{h}^{*}\right)+\frac{\sigma_{4}^{2} I_{h}^{*}}{2} \\
\leq & 2 \beta_{h}\left(S_{h}-S_{h}^{*}\right)^{2}+\beta_{h}\left(I_{a}-I_{a}^{*}\right)^{2}+\frac{\beta_{h} G\left(I_{a}^{*}\right)}{I_{h}^{*}}\left(I_{h}-I_{h}^{*}\right)\left(S_{h}-S_{h}^{*}\right) \\
& +2 \beta_{h} S_{h}^{* 2}+\beta_{h} I_{a}^{* 2}+\frac{\beta_{h} G^{2}\left(I_{a}^{*}\right)}{I_{h}^{*}}+\frac{\sigma_{4}^{2} I_{h}^{*}}{2} \\
= & 2 \beta_{h}\left(S_{h}-S_{h}^{*}\right)^{2}+\beta_{h}\left(I_{a}-I_{a}^{*}\right)^{2}+\frac{\beta_{h} G\left(I_{a}^{*}\right)}{I_{h}^{*}}\left(I_{h}-I_{h}^{*}\right)\left(S_{h}-S_{h}^{*}\right)+\eta_{4} .
\end{aligned}
$$

Now, define a $C^{2}$ function $V: R_{+}^{4} \rightarrow R_{+}$as follows

$$
V\left(S_{a}, I_{a}, S_{h}, I_{h}\right)=V_{1}+V_{2}+\frac{\beta_{h} G\left(I_{a}^{*}\right)}{I_{h}^{*}} V_{3}+\left(2 \mu_{h}+\delta_{h}+\gamma\right) V_{4} .
$$

Combining (17), (18), (19) and (20), we derive that

$$
\begin{aligned}
L V \leq & \left(-\frac{r_{a}}{k_{a}}+\frac{2+\sqrt{3}}{\sqrt{3}} \beta_{a}\right)\left(S_{a}-S_{a}^{*}\right)^{2} \\
& -\left[\mu_{a}+\delta_{a}-\sigma_{2}^{2}-\frac{4+\sqrt{3}}{\sqrt{3}} \beta_{a}-\beta_{h}\left(2 \mu_{h}+\delta_{h}+\gamma\right)\right]\left(I_{a}-I_{a}^{*}\right)^{2} \\
& -\left[\frac{\beta_{h} G\left(I_{a}^{*}\right)}{I_{h}^{*}}\left(\mu_{a}-\sigma_{3}^{2}\right)-2 \beta_{h}\left(2 \mu_{h}+\delta_{h}+\gamma\right)\right]\left(S_{h}-S_{h}^{*}\right)^{2} \\
& -\frac{\beta_{h} G\left(I_{a}^{*}\right)}{I_{h}^{*}}\left(\mu_{h}+\delta_{h}+\gamma-\sigma_{4}^{2}\right)\left(I_{h}-I_{h}^{*}\right)^{2}+\eta,
\end{aligned}
$$

where 


$$
\begin{aligned}
& \eta=\eta_{1}+\eta_{2}+\frac{\beta_{h} G\left(I_{a}^{*}\right)}{I_{h}^{*}}+\eta_{3}+\left(2 \mu_{h}+\delta_{h}+\gamma\right) \eta_{4}, \\
& \eta_{1}=\frac{\beta_{a} f^{2}\left(I_{a}^{*}\right)}{2}+\frac{3 \beta_{a} S_{a}^{* 2}}{2}+\frac{\sigma_{1}^{2} S_{a}^{*}}{2}+\beta_{a} I_{a}^{* 2}, \\
& \eta_{2}=\left(\sigma_{2}^{2}+\frac{4}{\sqrt{3}} \beta_{a}\right) I_{a}^{* 2}+\beta_{a} S_{a}^{*} I_{a}^{*} f\left(I_{a}^{*}\right)+\frac{2 \beta_{a}}{\sqrt{3}} S_{a}^{* 2}, \\
& \eta_{3}=\sigma_{3}^{2} S_{h}^{* 2}+\sigma_{4}^{2} I_{h}^{* 2}, \\
& \eta_{4}=2 \beta_{h} S_{h}^{* 2}+\beta_{h} I_{a}^{* 2}+\frac{\beta_{h} G^{2}\left(I_{a}^{*}\right)}{2}+\frac{\sigma_{4}^{2} I_{h}^{*}}{2} .
\end{aligned}
$$

It then follows from Theorem 6 in [21] that

$$
\begin{aligned}
& \limsup _{t \rightarrow \infty} \frac{1}{t} E \int_{0}^{t}\left(m_{1}\left(S_{a}(s)-S_{a}^{*}\right)^{2}+m_{2}\left(I_{a}(s)-I_{a}^{*}\right)^{2}\right. \\
& \left.+m_{3}\left(S_{h}(s)-S_{h}^{*}\right)^{2}+m_{4}\left(I_{h}(s)-I_{h}^{*}\right)^{2}\right) \mathrm{d} s \leq \eta .
\end{aligned}
$$

\section{Conclusion}

Most systems in the real world are disturbed by various stochastic factors, such as population mobility and meteorological factors including humidity, temperature and precipitation. Hence the effects of environmental fluctuation on the transmission of infectious diseases cannot be neglected. In this paper, we studied a stochastic avian-human influenza epidemic model with logistic growth for birds. To begin with, we proved the existence and uniqueness of global positive solution. For the stochastic avian-only system, set $R_{0}^{s}=\frac{\beta_{a} k_{a}}{\mu_{a}+\delta_{a}+\frac{\sigma_{2}^{2}}{2}}$, then the disease will tend to extinction if $R_{0}^{s}<1$, while system (5) will exist a unique ergodic stationary distribution if $R_{0}^{s}>\frac{2 r_{a}}{2 r_{a}-\sigma_{1}^{2}}$ and $0<\sigma_{2}^{2}<2\left(\mu_{a}+\delta_{a}\right)$. Hence it is interesting to note that a threshold number is difficult to obtain because of the logistic growth rate. For the full stochastic system, the disease will tend to extinction if $R_{0}^{s}<1$. From the viewpoint of biology, $R_{0}^{s}$ is a proper threshold parameter and the noise intensity in the infected avian population plays a key role. Moreover, we also discussed the asymptotic behavior and proved that the solution of the system (3) oscillates around corresponding endemic equilibrium under some conditions.

Some interesting topics deserve further consideration. For instance, it has not been confirmed that avian influenza virus does not spread from person to person and mutate. Furthermore, the seasonal effect for the transmission of avian influenza is neglected in the present model. We hope to study the comprehensive impacts of seasonal variation and environmental noises in the future.

\section{Acknowledgements}

We are very grateful to the editor and anonymous referees for their careful and 
valuable comments, which greatly improved the presentation of the paper. This work was supported by the National Natural Science Foundations of China (No. 11501364) and the Development Project of Science and Technology of University of Shanghai for Science and Technology (No. 2018KJFZ148 and No. 2019KJFZ171).

\section{Conflicts of Interest}

The authors declare no conflicts of interest regarding the publication of this paper.

\section{References}

[1] Liu, S., Ruan, S. and Zhang, X. (2017) Nonlinear Dynamics of Avian Influenza Epidemic Models. Mathematical Biosciences, 283, 118-135.

https://doi.org/10.1016/j.mbs.2016.11.014

[2] Centers for Disease Control and Prevention (CDC) (2017) First Human Infection with Avian Influenza A(H5N1) Virus Since Sept. Reported in Nepal. https://www.cdc.gov/flu/spotlights/2018-2019/h5n1-human-infection.html

[3] Alexander, D.J., Allan, W.H., Parsons, D. and Parsons, G. (1978) The Pathogenicity of Four Avian Influenza Viruses for Chickens, Turkeys and Ducks. Research in Veterinary Science, 24, 242-247. https://doi.org/10.1016/S0034-5288(18)33080-7

[4] Grais, R.F., Ellis, J.H. and Glass, G.E. (2003) Assessing the Impact of Airline Travel on the Geographic Spread of Pandemic Influenza. European Journal of Epidemiology, 18, 1065-1072. https://doi.org/10.1023/A:1026140019146

[5] Ferguson, N.M. and Cummings, D.A.T. (2005) Strategies for Containing an Emerging Influenza Pandemic in Southeast Asia. Nature, 437, 209-214. https://doi.org/10.1038/nature04017

[6] Menach, A.L. (2006) Key Strategies for Reducing Spread of Avian Influenza among Commercial Poultry Holdings: Lessons for Transmission to Humans. Proceedings of the Royal Society B, 273, 2467-2475. https://doi.org/10.1098/rspb.2006.3609

[7] Shi, Z., Zhang, X. and Jiang, D. (2019) Dynamics of an Avian Influenza Model with Half-Saturated Incidence. Applied Mathematics and Computation, 355, 399-416. https://doi.org/10.1016/j.amc.2019.02.070

[8] Iwami, S., Takeuchi, Y. and Liu, X. (2007) Avian-Human Influenza Epidemic Model. Mathematical Biosciences, 207, 1-25. https://doi.org/10.1016/j.mbs.2006.08.001

[9] Liu, S., Pang, L., Ruan, S. and Zhang, X. (2015) Global Dynamics of Avian Influenza Epidemic Models with Psychological Effect. Computational and Mathematical Methods in Medicine, 2015, 1-12. https://doi.org/10.1155/2015/913726

[10] Tuncer, N. and Martcheva, M. (2013) Modeling Seasonality in Avian Influenza H5N1. Journal of Biological Systems, 21, 1-30. https://doi.org/10.1142/S0218339013400044

[11] Liu, M., Huang, J., Ruan, S. and Yu, P. (2019) Bifurcation Analysis of an SIRS Epidemic Model with a Generalized Nonmonotone and Saturated Incidence Rate. Journal of Differential Equations, 267, 1859-1898.

https://doi.org/10.1016/j.jde.2019.03.005

[12] Zhang, Y., Zhang, L. and Yuan, S. (2018) The Effect of Media Coverage on Threshold Dynamics for a Stochastic SIS Epidemic Model. Physica A, 512, 248-260. 
https://doi.org/10.1016/j.physa.2018.08.113

[13] Liu, Q. and Jiang, D. (2017) Stationary Distribution and Extinction of a Stochastic SIR Model with Nonlinear Perturbation. Applied Mathematics Letters, 73, 8-15. https://doi.org/10.1016/j.aml.2017.04.021

[14] Meng, X., Zhao, S. and Feng, T. (2016) Dynamic of a Novel Nonlinear Stochastic SIS Epidemic Model with Double Epidemic Hypothesis. Journal of Mathematical Analysis and Applications, 433, 227-242. https://doi.org/10.1016/j.jmaa.2015.07.056

[15] Li, D., Cui, J., Liu, M. and Liu, S. (2015) The Evolutionary Dynamics of Stochastic Epidemic Model with Nonlinear Incidence Rate. Bulletin of Mathematical Biology, 77, 1705-1743. https://doi.org/10.1007/s11538-015-0101-9

[16] Zhang, F. and Zhang, X. (2018) The Threshold of a Stochastic Avian-Human Influenza Epidemic Model with Psychological Effect. Physica A, 492, 485-495. https://doi.org/10.1016/j.physa.2017.10.043

[17] Zhang, X. (2017) Global Dynamics of a Stochastic Avian Chuman inluenza Epidemic Model with Logistic Growth for Avian Population. Nonlinear Dynamics, 90, 2331-2343. https://doi.org/10.1007/s11071-017-3806-5

[18] Kutoyants, A.Y. (2003) Statistical Inference for Ergodic Diffusion Processes. Springer, London. https://doi.org/10.1007/978-1-4471-3866-2

[19] Ikeda, N. and Watanabe, S. (1997) A Comparison Theorem for Solutions Of Stochastic Differential Equations and Its Applications. Osaka. Journal of Mathematics, 14, 619-633.

[20] Has'minskii, R.Z. (1980) Stochastic Stability of Differential Equations. Sijthoff \& Noordhoff. Alphen aan den Rijn, The Netherlands.

[21] Kushner, H.J. (1967) Stochastic Stability and Control. Academic Press, New York. 